\title{
Violência sexual: características sociodemográficas de mulheres atendidas em um município do nordeste brasileiro
}

\author{
Sexual violence: sociodemographic characteristics of women served in a northeast \\ brazilian municipality \\ Violencia sexual: características sociodemográficas de las mujeres servidas en un \\ municipio brasileño del noreste
}

Audimere Monteiro Pereira ${ }^{1}$, Davydson Gouveia Santos ${ }^{2 \star}$, Evangelia Kotzias Atherino dos Santos ${ }^{2}$, Josiane Costa e Silva ${ }^{1}$, Marli Terezinha Stein Backes ${ }^{2}$, Yanna Gomes de Sousa ${ }^{3}$, Kalende das Misérias de Menezes Kalivala4, Caroline Bittelbrunn², Mailson Alagoneis Barbosa de Brito.

\section{RESUMO}

Objetivo: Identificar e descrever as características sociodemográficas de mulheres vítimas de violência sexual atendidas em um município do Nordeste Brasileiro. Métodos: Estudo descritivo, documental, retrospectivo, com abordagem quantitativa. A coleta dos dados foi realizada mediante dos formulários do Sistema de Informação de Agravos de Notificação, em dezembro de 2017. O período de abrangência compreendeu os anos de 2012 a 2017. Com estatística não paramétrica. Resultados: Foram avaliadas 287 notificações de violência sexual contra mulheres, onde $51,21 \%(n=147)$ desses casos ocorreram na cidade de Campina Grande, com faixa etária entre 12 a 18 anos com 44,25\% ( $n=127)$, eram mulheres solteiras $59,58 \%(n=171)$, estudantes 50,17\% ( $n=149)$, violentadas em via pública 49,82\% ( $n=128)$. Conclusão: Os dados do estudo revelam que as vítimas são predominantemente adolescentes jovens, solteiras e com baixo grau de escolaridade, com os casos ocorrendo em via pública no meio urbano, refletindo a falta de segurança pública que permeia todo país.

Palavras-chave: Delitos sexuais, Violência, Estupro, Mulheres, Notificação.

\begin{abstract}
Objective: To identify and describe the sociodemographic characteristics of women victims of sexual violence treated in a municipality in Northeast Brazil. Method: Descriptive, documentary, retrospective study with a quantitative approach. Data collection was performed using the forms of the Information System for Notifiable Diseases, in December 2017. The coverage period covered the years 2012 to 2017. With non-parametric statistics. Results: 287 notifications of sexual violence against women were evaluated, where $51,21 \%(\mathrm{n}=$ 147) of these cases occurred in the city of Campina Grande, aged 12 to 18 years with $44,25 \%(n=127)$, were single women $59.58 \%(n=171)$, students $50,17 \%(n=149)$, raped on public roads $49,82 \%(n=128)$. Conclusions: The study data reveal that the victims are predominantly young, single and low-educated adolescents, with the cases occurring on public roads in urban areas, reflecting the lack of security that permeates the whole country.
\end{abstract}

Keywords: Sexual offenses, Violence, Rape, Women, Notification.

\footnotetext{
${ }_{1}^{1}$ Faculdade de Campina Grande (UNESC), Campina Grande - PB.

2 Universidade Federal de Santa Catarina (UFSC), Florianópolis - SC.

*E-mail: davydson_gs@hotmail.com

${ }^{3}$ Universidade Federal do Rio Grande do Norte (UFRN), Natal - RN.

${ }^{4}$ Instituto Superior Politécnico da Caála, Huambo - Angola.
} 


\section{RESUMEN}

Objetivo: identificar y describir las características sociodemográficas de las mujeres víctimas de violencia sexual tratadas en un municipio del noreste de Brasil. Método: Estudio descriptivo, documental, retrospectivo con enfoque cuantitativo. La recopilación de datos se realizó utilizando los formularios del Sistema de Información para Enfermedades de Notificación, en diciembre de 2017. El período de cobertura abarcó los años 2012 a 2017. Con estadísticas no paramétricas. Resultados: se evaluaron 287 notificaciones de violencia sexual contra mujeres, donde $51,21 \%(n=147)$ de estos casos ocurrieron en la ciudad de Campina Grande, de 12 a 18 años con $44,25 \%(n=127)$, fueron mujeres solteras $59,58 \%(n=171)$, estudiantes $50,17 \%$ $(n=149)$, violadas en la vía pública $49,82 \%(n=128)$. Conclusiones: Los datos del estudio revelan que las víctimas son predominantemente adolescentes jóvenes, solteros y poco educados, con casos que ocurren en vías públicas en áreas urbanas, reflejando la inseguridad que permea todo el país.

Palabras-clave: Delitos sexuales, Violencia, Violación, Mujer, Notificación.

\section{INTRODUÇÃO}

A violência sexual é um problema de saúde pública universal, visualizando a gravidade e impacto na violação dos direitos humanos, como também nas múltiplas dimensões do ser humano, a exemplo de violação física, violação psíquica, e não menos importante, a violação social, tornou-se reconhecida como em 1993, pela Organização Pan-Americana da Saúde (OPAS) e Organização Mundial de Saúde (OMS). Desde então, ações foram implementadas para estimular estudos sobre a violência contra a mulher, propondo intervenções relativas à prevenção da agressão, atendimento normatizado, adequado e humanizado (KRUG EG, et al., 2002; UNITED, 1993).

Este fenômeno multidimensional afeta as cidadãs, independente da classe social, raça, etnia e orientação sexual, é caracterizado pelo ato de violação dos direitos humanos, restrigindo as mulheres no seu direito à vida, em toda amplitude da expressão, onde há privação da liberdade de opinião, sexualidade, saúde e à integridade física, este ameaçador fenômeno encontra-se presente no cotidiano das mulheres brasileiras (BRASIL, 2015).

Dentre as diversas formas de violência deparamos com uma das mais perversas, a violência sexual, que se encontra em um quadro de terror particular, silencioso e devastador, capaz de promover desequilíbrios físicos, psicológicos e sociais à mulher vítima de abuso sexual. A vítima dessa modalidade de violência pode apresentar isolamento psicológico, social, sentimentos negativos, dificuldades nas relações interpessoais evidenciados pelo medo e reprovação, além de baixa autoestima, sentimentos repugnantes de si mesmo, desprestígio da sociedade, até a incapacidade de enfrentar essas situações como pessoa e cidadã (DELZIOVO CR, et al., 2018).

Diante dessa problemática, observa-se a importância da sensibilização dos profissionais de saúde, principalmente, por parte da enfermagem, que atuam na porta de entrada dos serviços de saúde que atendem às mulheres vítimas de violência. A equipe precisa está preparada para identificar as necessidades da mulher violentada, acolher a vítima e saber tratá-la por meio de protocolos destinados à temática e referenciá-las, se necessário (KATAGUIRI LG, et al., 2019). Os profissionais devem agir, de forma cautelosa e respeitosa, tendo em vista a fragilidade da mulher nessa situação, orientando as mulheres vítimas de agressão quanto à rede intra e intersetorial que existe com o objetivo de protegê-las. Os profissionais devem estar atentos também, a identificação correta das vítimas e notificação dos casos e esses setores devem agir de forma integrada para que a vítima tenha uma assistência humanizada, completa e de qualidade (BATISTETTI L, et al., 2020).

De acordo com as considerações apresentadas, destaca-se a importância dos serviços de saúde em resposta à violência sexual contra as mulheres, pois muitas vezes é o primeiro local onde as vítimas buscam atendimento e a conscientização dos profissionais de saúde é fundamental, enfatizado pela humanização do acolhimento e atendimento, propiciando que a mulher vítima de abuso sexual, tenha um atendimento resolutivo e integral, além da importância das notificações dos casos suspeitos e confirmados (GARCIA LP, 2016). 
A violência sexual dirigida às mulheres é um problema de saúde pública no país tendo em vista o grande número de casos e esse estudo busca dar mais visibilidade a esse tipo de violência, além de propiciar o conhecimento da assistência à saúde no serviço de referência, no sentido de manter e ou readequar as práticas desenvolvidas visando à integralidade do cuidado. Além disso, pesquisas com esta temática retratam o que deve ser mudado e os desafios futuros para a obtenção da qualidade do serviço ofertado a vítimas de violência sexual (TRENTIN D, et al., 2019).

Objetiva-se com o estudo identificar e descrever as características sociodemográficas de mulheres vítimas de violência sexual atendidas em uma maternidade referência de um município do Nordeste Brasileiro.

\section{MÉTODOS}

Trata-se de um estudo descritivo, documental, retrospectiva, com abordagem quantitativa. $O$ estudo foi realizado em uma instituição hospitalar no interior do Estado da Paraíba, referência para atendimento às mulheres em situação de violência sexual para os municípios pertencentes à segunda macrorregião do Estado.

A instituição hospitalar é referência para o município e cidades vizinhas em atenção à saúde da mulher gestante e recém-nascidos, contando com setor de planejamento familiar, pré-natal de alto risco, triagem, classificação de risco, sala de parto, centro cirúrgico, alojamento conjunto, UTI obstétrica, UTI Neonatal, projeto mãe canguru, triagem neonatal, banco de leite humano, entre outros que asseguram o atendimento de qualidade as pacientes que necessitam dos serviços 24 horas por dia, constituído de equipe multidisciplinar.

Esta instituição é responsável em receber as mulheres em situação de violência sexual para atendimento inicial, composta pelo exame físico genital (quando necessário para possível tratamento cirúrgico); tratamento farmacológico profilático para possíveis Infecções Sexualmente Transmissíveis (IST's) e administração da pílula anticoncepcional oral quando necessário.

A amostra do estudo foi composta pelas fichas do Sistema de Informação de Agravos de Notificação (SINAN) disponíveis no hospital de referência para assistência às mulheres em situação de violência sexual. Foram incluídos na pesquisa todos os formulários do SINAN das mulheres atendidas de janeiro de 2012 a novembro de 2017.

Formulários rasurados, danificados ou que apresentassem qualquer ilegibilidade que impossibilitasse a coleta dos dados foram excluídos, sendo estes os critérios de exclusão. A amostra resultante foi de 287 formulários que atenderam aos critérios de inclusão.

A coleta dos dados foi realizada na primeira semana de dezembro de 2017, sem dificuldade para o acesso aos formulários. Por meio do instrumento de coleta utilizou-se de variáveis como o ano e local do estupro; idade, situação conjugal, nível de escolaridade, ocupação das vítimas; quanto ao agressor, observou-se o número de envolvidos, zona de atuação, uso de álcool pelo agressor e o meio de agressão: como forma de abordagem da vítima. Estes documentos foram importantes, uma vez que contribuíram com os dados sociais das pacientes atendidas, para análise e elaboração dos resultados.

Após a coleta, os dados foram registrados em planilha do software Microsoft Excel 16.0 (Office 2016), desempenhando a técnica de dupla digitação. Com a garantia da digitação correta dos dados foram exportados e analisados pelo programa Statistical Package for Social Science (SPSS), versão SPSS Student. Foi considerado um intervalo de confiança de $95 \%$ diante da amostra do estudo.

O estudo foi submetido ao Comitê de Ética e Pesquisa (CEP) da Universidade Estadual da Paraíba (UEPB) via Plataforma Brasil, para apreciação e recebeu parecer favorável à sua realização sob número CAAE: 77377717.5.0000.5187 com o Parecer Consubstanciado no 2.343.253. O estudo cumpriu integralmente as normas da resolução ํㅜ $466 / 12$ do Conselho Nacional de Saúde e suas complementares. 


\section{RESULTADOS}

Entre 2012 a novembro de 2017 houve 287 casos notificados de violência sexual no município de Campina Grande, porém, esse número abrange casos ocorridos nas cidades vizinhas, que buscam o serviço por ser referência à atenção à saúde da mulher, agravo relacionado à violência doméstica e sexual, bem como as questões reprodutivas e sexuais da mulher.

O serviço designado, Centro de Referência à Saúde da Mulher, responsável pelo acolhimento e atendimento médico, psicológico e social, orientação e encaminhamento jurídico à mulher em situação de violência, atuam desenvolvendo atendimento e o acolhimento necessário à superação de situação de violência, contribuindo para o fortalecimento da mulher, garantindo estabilidade tanto clínica como emocional e o resgate de sua cidadania. A Tabela 1 expressa o número de casos notificados por ano, entre os anos deste estudo, destaca-se o ano de 2012 por ter sido o ano de implantação do serviço, com a possibilidade de uma elevada taxa de subnotificações.

Tabela 1 - Números de casos notificados por ano, no período de 2012 a 2017. Campina Grande-PB, 2017.

\begin{tabular}{ccc}
\hline Ano Notificado & $\mathbf{n} \times$ de casos & $\%$ \\
\hline 2012 & 36 & 12,54 \\
2013 & 43 & 14,98 \\
2014 & 41 & 14,28 \\
2015 & 39 & 13,60 \\
2016 & 76 & 26,48 \\
2017 & 52 & 18,12 \\
\hline Total & $\mathbf{2 8 7}$ & $\mathbf{1 0 0}$ \\
\hline
\end{tabular}

Fonte: PEREIRA AM, et al., 2017.

Com a apresentação do número de casos notificados por ano, identifica-se que $51,21 \%$ ( $n=147)$ das notificações são de mulheres oriundas do município de Campina Grande e 8,36\% ( $n=24)$ são do município de Queimadas. Municípios com 15km de distância. Observa-se na tabela 2 a distribuição dos casos pelos municípios atendidos no centro de referência regional.

Tabela 2 - Distribuição de casos por município notificados em Campina Grande. Campina Grande-PB, 2017.

\begin{tabular}{lcc}
\hline Local da Ocorrência & no de casos & $\%$ \\
\hline Campina Grande & 147 & 51,21 \\
Queimadas & 24 & 8,36 \\
Esperança & 14 & 4,90 \\
Lagoa Seca & 11 & 3,84 \\
Fagundes & 09 & 3,13 \\
Alagoa Grande & 09 & 3,13 \\
Pocinhos & 07 & 2,43 \\
Barra de Santana & 04 & 1,40 \\
Aroeiras & 04 & 1,40 \\
Outros Municípios & 58 & 20,20 \\
\hline Total & $\mathbf{2 8 7}$ & $\mathbf{1 0 0}$
\end{tabular}

Fonte: PEREIRA AM, et al., 2017.

O perfil sociodemográfico das mulheres vítimas de violência sexual é apresentado na Tabela 3 seguindo as variáveis pertinentes ao estudo. 
Tabela 3 - Distribuição das mulheres agredidas sexualmente de acordo com algumas variáveis. Campina Grande-PB, 2017.

\begin{tabular}{|c|c|c|c|}
\hline & Variáveis & $\mathbf{N}$ & $\%$ \\
\hline \multirow{6}{*}{ Faixa Etária } & $0-10$ anos & 15 & 5,22 \\
\hline & $11-18$ anos & 127 & 44,25 \\
\hline & $19-30$ anos & 97 & 33,80 \\
\hline & $31-50$ anos & 37 & 12,90 \\
\hline & $51-89$ & 11 & 3,83 \\
\hline & Total & 287 & 100 \\
\hline \multirow{5}{*}{ Situação Conjugal } & Solteiras & 171 & 59,60 \\
\hline & Casadas & 48 & 16,72 \\
\hline & Viúvas & 10 & 3,48 \\
\hline & Não Registrado/ignorado & 58 & 20,20 \\
\hline & Total & 287 & 100 \\
\hline \multirow{10}{*}{ Escolaridade } & Analfabeta & 15 & 5,22 \\
\hline & Alfabetizada & 47 & 16,40 \\
\hline & Ensino fundamental incompleto & 20 & 6,96 \\
\hline & Ensino fundamental completo & 07 & 2,44 \\
\hline & Ensino médio incompleto & 42 & 14,63 \\
\hline & Ensino médio completo & 15 & 5,22 \\
\hline & Ensino superior incompleto & 08 & 2,78 \\
\hline & Ensino superior completo & 06 & 2,10 \\
\hline & Não registrado/ignorado & 127 & 44,25 \\
\hline & Total & 287 & 100 \\
\hline \multirow{5}{*}{ Ocupação } & Estudante & 149 & 51,91 \\
\hline & Doméstica & 53 & 18,46 \\
\hline & Outras Profissões & 34 & 11,85 \\
\hline & Não registrado/ignorado & 51 & 17,78 \\
\hline & Total & 287 & 100 \\
\hline \multirow{4}{*}{ Local da agressão } & Via pública & 128 & 44,60 \\
\hline & Residência & 109 & 37,98 \\
\hline & Outros & 50 & 17,42 \\
\hline & Total & 287 & 100 \\
\hline \multirow{4}{*}{ Zona de atuação } & Urbana & 143 & 49,83 \\
\hline & Rural & 41 & 14,28 \\
\hline & Não registrado/ignorado & 103 & 35,89 \\
\hline & Total & 287 & 100 \\
\hline
\end{tabular}

Fonte: PEREIRA AM, et al., 2017.

A identificação do perpetrador contribui para categorizar o perfil do agressor deste tipo de violência. A Tabela 4 traz algumas características dos perpetradores identificadas pelas mulheres no momento da violência sexual. 
Tabela 4 - Características dos perpetradores segundo as mulheres agredidas. Campina Grande-PB, 2017.

\begin{tabular}{llll}
\hline & Variáveis & $\mathbf{N}$ & $\%$ \\
\hline \multirow{3}{*}{ Tipo de perpetrador } & Desconhecido da vítima & 164 & 57,15 \\
& Conhecido da vítima & 123 & 42,85 \\
\cline { 2 - 4 } & Total & $\mathbf{2 8 7}$ & $\mathbf{1 0 0}$ \\
\hline \multirow{3}{*}{ No de perpetradores } & Um & 253 & 88,15 \\
& Dois & 22 & 7,67 \\
& Mais de dois & 12 & 4,18 \\
\cline { 2 - 4 } Uso de álcool ou outras drogas & Total & $\mathbf{2 8 7}$ & $\mathbf{1 0 0}$ \\
\multirow{2}{*}{ pelo perpetrador } & Sim & 78 & 27,18 \\
& Não & 135 & 47,04 \\
& Não registrado/ignorado & 74 & 25,78 \\
\cline { 2 - 4 } Meio de agressão & Total & $\mathbf{2 8 7}$ & $\mathbf{1 0 0}$ \\
& Ameaça & 185 & 64,45 \\
& Força física/enforcamento & 151 & 52,61 \\
& Arma de fogo & 32 & 11,14 \\
\hline
\end{tabular}

Fonte: PEREIRA AM, et al., 2017.

\section{DISCUSSÃO}

Nesse estudo observou-se que em 2012, foram 36 casos confirmados de violência sexual contra a mulher, acredita-se numa elevada taxa de subnotificação devido ao processo de implantação do serviço, e a organização do setor de epidemiologia que ocorreu neste ano em questão. A subnotificação possui sérias implicações, tais como a impunidade do agressor, a falta de conhecimento dos profissionais e da própria população acerca do serviço e a ausência de mobilização social. A violência é incluída na relação de doenças e agravos de notificação compulsória em todo o território nacional desde 2011 (BRASIL, 2011).

Em contrapartida, observou-se um aumento significativo no número de casos notificados no ano de 2016 , pode-se atribuir esse resultado à organização do serviço, a maior conscientização da população sobre a importância de denunciar a violência, e dos profissionais de saúde em notificar esse agravo, como relatam estudos anteriores que descrevem à organização dos serviços de vigilância mais sensível à identificação dos casos (LEITE AC e FONTANELLA BJB, 2019; SILVA LEL e OLIVEIRA MLC, 2016).

A violência sexual, embora seja um fenômeno de grande magnitude e impacto negativo a saúde da mulher, permanece oculta na sociedade, pois ainda é reduzido o número de vítimas que realizam a denúncia. Tratase do medo de retaliação por parte do agressor (DELZIOVO CR, et al., 2017). Este é um fato agravante, devido aos reduzidos dados estatísticos reais relacionados à violência sexual, o que tende a minimizar ações referentes ao poder público a frente desse agravo considerando que, de acordo com a literatura, a violência, em especial a do tipo sexual no mundo tem elevada subnotificação. Lembrando que, com a notificação, além de interromper o ciclo de violência, viabiliza formas de promoção e de prevenção que levem em conta as especificidades culturais das várias regiões do país.

Em relação às notificações do ano de 2016, dos 76 casos notificados e confirmados, $51,31 \%$ ( $n=39)$ deles, foi de casos de violência sexual ocorridos na cidade de Campina Grande, o que caracteriza a insegurança e a vulnerabilidade das mulheres. É necessário a manutenção e desenvolvimento de políticas públicas destinadas a essa demanda, a identificação dos serviços importantes no combate à violência com ênfase na questão de segurança e a da Lei Maria da Penha que apesar de suas fragilidades confere proteção legal à mulher visando coibir a violência (GASPAR RS e PEREIRA MUL, 2018; BRASIL, 2006).

De acordo com a Lei 11.340/2006, Lei Maria da Penha, que tem se destacado em relação ao combate a violência doméstica e familiar, garantindo a punição com maior rigor dos agressores, fazendo com que 0 ato de violência destinado às mulheres, deixe de ser interpretada como um problema individual da mulher violentada, passando a ser visto como problema social e do estado que deve uma série de ações políticas, sanções legais e assistência social a fim de minimizar a violência contra as mulheres (BRASIL, 2006). 
Verificou-se no presente estudo que o município de Campina Grande apresenta 51,21\% ( $n=147)$ de todas as notificações, seguido pelo município de Queimadas com $8,36 \%(n=24)$ dos casos já com uma grande diferença entre o número dos casos que ocorreram em municípios vizinhos. O que chama a atenção para o grande número de notificações referente ao município de Campina Grande em relação às demais cidades é a existência do crescimento urbano acelerado, que traz um grande fluxo de pessoas para as áreas urbanas e assim contribui para um crescimento desordenado e desorganizado das cidades, sem nenhum planejamento prévio. Quanto ao aumento de casos de violência, isso é reflexo de políticas de segurança pública voltada apenas para a questão da repressão ao invés de tratar a questão social (ARRUDA BB e SANTOS JAC, 2019).

Referente à idade das vítimas no momento da agressão sexual, observa-se que $44,25 \%$ ( $n=127)$ das mulheres agredidas sexualmente encontram-se na faixa etária de 11 a 18 anos, os resultados desta pesquisa corroboram com os dados apresentados por outras investigações. Estudo realizado no hospital universitário da capital paranaense, referência para vítimas de violência, mostra a vulnerabilidade das adolescentes jovens a frente dessa questão, revelando que o maior número de atendimentos ocorreu na faixa etária de 12 aos 18 anos, totalizando $53,46 \%$ ( $n=680)$ dos casos do estudo (TRIGUEIRO TH, et al., 2015).

Outro estudo que realizou o mapeamento da violência sexual no Brasil, mostra que $11,9 \%$ dos casos de violência contra a mulher são do tipo sexual, destacando a vulnerabilidade feminina, a maior prevalência foi maior entre as adolescentes com idade entre 12 e 17 anos com 24,3\% casos (WAISELFISZ JJ, 2015).

A violência praticada contra a mulher deixa marcas profundas principalmente de ordem psicológica, uma em cada três ou quatro meninas é abusada sexualmente antes de completar 18 anos, o que indica também maior vulnerabilidade destas ao tráfico e à exploração sexual, $49.2 \%$ dos agressores reincidem na agressão (WAISELFISZ JJ, 2015). Observa-se que muito da violência causada a esse grupo é feita por parceiro íntimo desde a adolescência e que por vezes perdura até a vida adulta, o enfrentamento da violência é entendido pelos profissionais do terceiro setor como de responsabilidade da área da saúde (LOURENÇO RG e FONSECA RMGS, 2020).

A tipificação da agressão contra as mulheres, viabiliza a criação de ações preventivas a violência e agravos de acordo com o tocante da vulnerabilidade feminina (SILVA MS, et al., 2020). Por vezes percebe-se a não resolutividade de ações de caráter social e as ações dos profissionais da saúde centradas no caráter biológico. Quanto à situação conjugal das mulheres atendidas em situação de violência sexual a predominância foi nas mulheres solteiras, com 59,60\% ( $n=171)$ dos casos, reforçando a ideia de vulnerabilidade. Esta vulnerabilidade é justificada pela possível ausência de relações de proteção, onde o perpetrador visualiza uma relação de domínio e poder sobre a sexualidade da mulher (MOREIRA KFA, et al., 2020; DELZIOVO CR, et al., 2018).

Em contraponto, mulheres casadas que são violentadas sexualmente muitas vezes são agredidas pelo próprio parceiro íntimo, aumentando o risco de contrair Infecções Sexualmente Transmissíveis devido a limitada capacidade de negociar o sexo seguro, pois esses parceiros possuem comportamentos de risco, tais como atividade sexual com outras parceiras sem preservativo e uso de drogas (ALEXANDER KA, 2020).

Quanto à ocupação das mulheres observou-se que $51,91 \%(n=149)$ são estudantes e $17,42 \%$ (n=50) são domésticas; e que as agressões ocorrem em via pública $45,59 \%$ ( $n=128)$ quanto nas residências $37,97 \%$ $(n=109)$ delas. Estudo realizado no estado de São Paulo, onde mostra que aproximadamente $54 \%$ dos eventos violentos foram perpetrados em vias públicas e $46 \%$ na residência da vítima (OSHIKATA CT, et al., 2011).

Esses dados vão de encontro com o que é descrito na obra literária "O que é violência contra a mulher", onde afirma que a violência contra à mulher se manifesta de diversas formas e ocorre tanto no âmbito familiar, como em locais públicos ou privados, no trabalho ou na própria comunidade (TELES MAA e MELO M, 2017).

Nesse estudo a agressão sexual está presente com maior frequência na zona urbana com 49,83\% ( $n=143)$ dos casos, que pode ser relacionado pela necessidade da realização das atividades diárias de trabalho, estudo e rotineiras no dia-dia, demonstrando uma maior vulnerabilidade das mulheres nos centros urbanos. O gênero sexual dos perpetradores foi em 100\% ( $n=287$ ) dos casos do sexo masculino neste estudo, sendo 
que em $11,85 \% \quad(n=24)$ dos casos ocorreram com dois ou mais perpetradores ao mesmo tempo, demonstrando um elevado número diante da amostra total. Verificou-se que em $57,15 \%(n=164)$ dos casos os agressores eram desconhecidos das vítimas, $42,85 \% \quad(n=123)$ dos casos os perpetradores eram conhecidos da vítima, não necessariamente com parentesco familiar.

Estudos semelhantes revelam variações quanto à identificação do perpetrador, onde é revelado que 43,5\% dos perpetradores eram pessoas desconhecidas da vítima, favorecendo a continuidade e consequente aumento de ocorrências pela falta de identificação (ACOSTA DF, et al., 2016). Divergindo de estudo realizado no Estado de Santa Catarina, onde $51,3 \%$ dos casos de estupros são praticados por conhecidos das vítimas (BAIGORRIA J, et al., 2017).

O uso de bebidas alcóolicas ou outras drogas por parte do perpetrador não é um fator determinante para impulsionar ou justificar tal ato de violência sexual as mulheres, mesmo com ausência de informações nos registros quanto a esse fator, ainda assim, os registros mostram que em $47,4 \% \quad(n=135)$ dos casos, os perpetradores não aparentavam terem feito uso de bebidas alcoólicas ou outras drogas. O uso de substâncias como o álcool pode desencadear o comportamento violento, porém, seu uso pode ser um fator antecipador e agravante de violência, mas não a causa primária da mesma (CARDOSO RBN e MOURA LBA, 2017).

A forma de abordagem de uma vítima para outra não é muito diferente, onde o agressor usa-se da fragilidade e vulnerabilidade da vítima, impondo sua força física, juntamente com a prática de enforcamento em $52,61 \%$ ( $n=151)$ dos casos; a coação, intimação e medo ocorreram em $64,45 \%$ ( $n=185)$ dos casos; o uso de arma de fogo foi registrado em $11,14 \%(n=32)$ dos casos registrados e objetos perfuro-cortantes em $8,01 \%$ $(n=23)$ dos casos na abordagem à mulher, é importante fazer o registro de que essas abordagens não ocorrem de forma isolada, logo, o perpetrador ao abordar a mulher associa mais de um meio de intimidação.

Observa-se o aumento do uso da força física, seguido da diminuição do uso de arma de fogo para intimidação, um fator que pode justificar essa mudança é a diminuição da circulação das armas de fogo após o referendo de 2005 no Brasil, causando aumento da violência contra à mulher praticada em seu próprio domicilio, através da coação, chantagem emocional e força física (OSHIKATA CT, et al., 2011; CARNEIRO JB, et al., 2020).

\section{CONCLUSÃO}

O estudo permite identificar e descrever as características sociodemográficas de mulheres vítimas de violência sexual atendidas em uma maternidade referência de um município do Nordeste Brasileiro. Os dados do estudo revelam que as vítimas são predominantemente adolescentes jovens, solteiras e com baixo grau de escolaridade, com os casos ocorrendo em via pública no meio urbano, refletindo a falta de segurança pública, que não é um problema regional, mas de âmbito nacional. Evidencia-se a necessidade do desenvolvimento de estratégias eficazes a fim de minimizar a vulnerabilidade das mulheres em relação à violência, como também a formulação e ampliação de políticas públicas baseadas na transversalidade do gênero.

\section{REFERÊNCIAS}

1. ACOSTA DF, et al. Violência sexual: da denúncia à criminalização do agressor [Sexual violence: from complaint to criminalization of offenders]. Revista Enfermagem UERJ, v. 24, n. 2, 2016.

2. ALEXANDER KA. Social Determinants of HIV/AIDS and Intimate Partner Violence: Interrogating the Role of Race, Ethnicity, Skin Color Rev. Latino-Am. Enfermagem. 2020;28:e3280

3. ARRUDA BB, SANTOS JAC. O enfrentamento da violência contra as mulheres sob o enfoque da justiça restaurativa e da perspectiva sistêmica. In Baggenstoss GA, Dos Santos PR, Sommariva SS, Hugill MSG. Não há lugar seguro. Florianópolis: Editora Centro de Estudos Jurídicos (CEJUR), 2019. P. 178 - 197.

4. BAIGORRIA J, et al. Serviço social e atenção às mulheres em situação de violência sexual: Experiência de um Hospital Geral. 2017.

5. BATISTETTI L, et al. The perception of sexual violence's victims about the embracement in a reference hospital in Paraná / A percepção da vítima de violência sexual quanto ao acolhimento em um hospital de referência no Paraná. Revista de Pesquisa: Cuidado é Fundamental Online [Internet]. 2020; 12(0): 169-175. 
6. BRASIL. Lei Maria da Penha. Lei n. 11.340/2006. Coíbe a violência doméstica e familiar contra a mulher. Presidência da República, 2006.

7. BRASIL MS. Secretaria de Atenção à Saúde. Departamento de Ações Programáticas Estratégicas. Aspectos Jurídicos do Atendimento às Vítimas de Violência Sexual. 2.ed. Brasília. 2011.

8. BRASIL MS. Secretaria de Vigilância em Saúde. Manual instrutivo de preenchimento da ficha de notificação/investigação individual violência doméstica, sexual e/ou outras violências. Brasília, 2015

9. CARDOSO RBN, MOURA LBA. Homens autores de violência contra parceiros íntimos: estudo com policiais militares do distrito federal. Anais, p. 1-21, 2017.

10. CARNEIRO JB, et al. Desvelando as estratégias de enfrentamento da violência conjugal utilizadas por mulheres. Texto contexto - enferm. [Internet]. 2020; v 29.

11. DELZIOVO CR, et al. Características dos casos de violência sexual contra mulheres adolescentes e adultas notificados pelos serviços públicos de saúde em Santa Catarina, Brasil. Cad. Saúde Pública [Internet]. 2017; 33(6): e00002716.

12. DELZIOVO CR, et al. Violência sexual contra a mulher e o atendimento no setor saúde em Santa Catarina - Brasil. Ciênc. Saúde coletiva [Internet]. 2018; 23(5): 1687-1696.

13. GARCIA LP. A magnitude invisível da violência contra a mulher. Epidemiol. Serv. Saúde [Internet]. 2016; 25(3): 451454.

14. GASPAR RS, PEREIRA MUL. Evolução da notificação de violência sexual no Brasil de 2009 a 2013. Cadernos de Saúde Pública [online]. 2018, v. 34, n. 11, e 00172617.

15. KATAGUIRI LG, et al. Caracterização da violência sexual em um estado da região sudeste do brasil. Texto contexto - enferm. [Internet]. 2019; 28: e20180183.

16. KRUG EG, et al., editors. World report on violence and health. Geneva: World Health Organization; 2002

17. LEITE AC, FONTANELLA BJB. Violência doméstica contra a mulher e os profissionais da APS: Predisposição para abordagem e dificuldades com a notificação. Rev Bras Med Fam Comunidade [Internet]. 2019; 14(41): 2059.

18. LOURENÇO RG, FONSECA RMGS. Primary Health Care and the Third Sector in the face of violence between intimate adolescent partners. Rev. Latino-Am. Enfermagem. 2020;28:e3341.

19. MOREIRA KFA, et al. Violência sexual contra mulheres em idade fértil na região norte do Brasil. REAS [Internet]. 2020;12(3): e2826.

20. OSHIKATA CT, et al. Características das mulheres violentadas sexualmente e da adesão ao seguimento ambulatorial: tendências observadas ao longo dos anos em um serviço de referência em Campinas, São Paulo, Brasil. Cad. Saúde Pública [Internet]. 2011; 27(4): 701-713.

21. SILVA LEL, OLIVEIRA MLC. Características epidemiológicas da violência contra a mulher no Distrito Federal, 2009 a 2012. Epidemiol. Serv. Saúde [Internet]. 2016; 25(2): 331-342.

22. SILVA MS, et al. Nursing care for child/adolescent victims of violence: integrative review / Assistência de enfermagem à criança/adolescente vítima de violência: revisão integrativa. Revista de Pesquisa: Cuidado é Fundamental Online [Internet]. 2020 Jan 9; 12(0): 115-123.

23. TELES MAA, MELO M. O que é violência contra a mulher. Brasiliense, 2017.

24. TRENTIN D, et al. Olhar de profissionais no atendimento a mulheres em situação de violência sexual: perspectiva da declaração universal de bioética e direitos humanos. Texto contexto - enferm. [Internet]. 2019; 28: e20180083.

25. TRIGUEIRO TH, et al. Vítimas de violência sexual atendidas em um serviço de referência. Cogitare Enfermagem, 2015 Abr/jun; 20(2):249-56.

26. UNITED NATIONS GENERAL ASSEMBLY. Declaration on the elimination of violence against women. Geneva: United Nations; 1993.

27. WAISELFISZ JJ. Mapa da violência 2015: Homicídio de mulheres no Brasil. Brasília, DF: FLACSO, 2015. 79 p. 